\title{
Design, synthesis and evaluation of 1,4-benzodioxine derivatives as novel platelet aggregation inhibitors
}

\author{
Zhouling Xie*,1, Lulu Zhao ${ }^{2}$, Xue Ding ${ }^{3}$, Yi Kong ${ }^{3}$ \& Zhiyu Li**,2 \\ ${ }^{1}$ School of Biological \& Medical Engineering, Hefei University of Technology, Hefei, Anhui 230009, PR China \\ ${ }^{2}$ Jiangsu Key Laboratory of Drug Design \& Optimization, China Pharmaceutical University, 24 Tongjiaxiang, Nanjing 21009, PR \\ China \\ ${ }^{3}$ School of Life Science \& Technology, China Pharmaceutical University, 24 Tongjiaxiang, Nanjing 210009, PR China \\ * Author for correspondence: zhoulingxie@hfut.edu.cn; xz13817@126.com \\ ** Author for correspondence: zhiyuli@cpu.edu.cn
}

Aim: To find novel platelet aggregation inhibitors, two new series of 1,4-benzodioxine derivatives were synthesized and screened for the ability to inhibit platelet aggregation. Materials \& methods: The synthesized compounds were evaluated for antiplatelet aggregation activity using human blood platelet and GPIIb/IIla antagonistic activity. Results: Compound 9-2p showed significant antiplatelet activity with the $I C_{50}$ values of 41.7 and $22.2 \mu \mathrm{M}$ induced by ADP and thrombin, respectively, more potent than that of LX2421. Compound 9-2p exhibited GPIlb/Illa antagonistic activity with the $\mathrm{IC}_{50}$ value of $2.3 \mu \mathrm{M}$, as potent as RGDs. In vivo study showed that 9-2p displayed remarkable antithrombotic activity, more effective than LX2421, but less effective than tirofiban. Conclusion: Compound 9-2p showed moderate antiplatelet activity and antithrombotic activity, which could be further optimized based on the target of GPIIb/IIla.

First draft submitted: 10 July 2017; Accepted for publication: 16 November 2017; Published online:

30 January 2018

Keywords: 1,4-benzodioxine $\bullet$ antiplatelet activity $\bullet$ antithrombotic activity • GPIlb/llla • GPIlb/Illa antagonist • thrombin

Arterial thrombosis is the most important determinant leading to arterial thromboembolic diseases such as myocardial infarction, unstable angina and cerebral ischemia, which are responsible for elevated mortality worldwide [1]. The adhesion, activation and aggregation of platelets display critical roles in hemostasis and its pathophysiology at the site of atherosclerotic lesions in coronary arteries. Following the interaction of collagen, ADP, thrombin and other platelet agonists released by vascular damage, platelets will be activated to be 'cross-link' status, which facilitates the formation of platelet-dependent thrombus $[2,3]$. Therefore, antiplatelet aggregation therapies targeting the key pathways of platelet activation have an important role in the treatment of thrombotic disorders. Currently, the most common of antiplatelet agents are represented as aspirin with the mechanism of blocking thromboxane A2 synthesis; clopidogrel with the mechanism of interfering ADP-mediated signaling and GPII/IIIa antagonists (e.g., tirofiban) with the mechanism of inhibiting GPII/IIIa binding to the adhesive protein fibrinogen [4-7].

Recent advances in antiplatelet therapy have remarkably reduced the risk of recurrent ischemic events in patients with acute coronary syndrome or undergoing percutaneous coronary intervention [4]. However, there remains a substantial incidence of arterial thrombosis in patients because of current therapy limitations. For aspirin, it shows weak inhibition of platelet function and 'resistant' in some patients; for clopidogrel, it only blocks one pathway of ADP-mediated signaling and displays slow onset of action; for GPIIb/IIIa antagonists, it cannot be treated by oral, fails to separate a reduction in thrombotic events from an increase in bleeding risk. Moreover, GPIIb/IIIa antagonists result in thrombocytopenia due to the exposure of ligand-induced binding sites induced by the conformational changes in GPIIb/IIIa. Therefore, it is of great interest to explore new agents, not only for use as drugs but also because such compounds could be used as pharmacological tools to provide important information regarding platelet function [8-11]. 


\section{Materials \& methods}

Synthesis

All NMR was recorded on Bruker AV-300 apparatus in deuterated solvents with tetramethylsilane as internal standard. High-resolution MS (HRMS) was collected on Agilent technologies 6520 Accurate-Mass Q-TOF LC/MS instruments. Every targeted compound was purified via silica gel (60 A, 70-230 mesh) column chromatography. Melting points were measured by XT- 4 melting point apparatus. The purity $(>95 \%)$ of final compounds is verified by the HPLC study performed on Agilent C18 $\left(4.6 \times 150 \mathrm{~mm}^{2}, 3.5 \mu \mathrm{m}\right)$ column using a mixture of solvent methanol/water at the flow rate of $0.5 \mathrm{ml} / \mathrm{min}$ and peak detection at $254 \mathrm{~nm}$ under UV. See the Supplementary Information for the synthesis details.

\section{Biology}

Human blood samples \& washed platelet preparation

Human platelet-rich plasma (PRP) was obtained by purchasing apheresis platelets from Jiangsu Province Blood Center (Nanjing, PR China). Washed platelets were prepared as described previously [12].

\section{Antiplatelet aggregation assay [13]}

Platelet aggregation was measured at $37^{\circ} \mathrm{C}$ in a platelet aggregometer (PRECILBY-NJ4, Pu Lisheng Corp. Beijing, PR China). Platelet-poor plasma was prepared by centrifugation at $3000 \times g$ for $10 \mathrm{~min}$ from PRP, removed with a pipette and used in the reference cell of the aggregometer. Test substances (dissolved in saline or $0.2 \%$ aqueous DMSO) were added to PRP to a final volume of $300 \mu \mathrm{l}$ and allowed to incubate for $1 \mathrm{~min}$ followed by the addition of an inducer (10 $\mu \mathrm{M}$ ADP or $0.33 \mathrm{U} / \mathrm{ml}$ thrombin) to initiate aggregation. The maximal gradient of platelet aggregation was defined as the maximal percent increase within the first $5 \mathrm{~min}$. The data were obtained in triplicates. The $\mathrm{IC}_{50}$ value was calculated as the concentration of inhibitor causing a $50 \%$ inhibition of the aggregation using SPSS software with at least four concentrations.

\section{Enzyme assay for inhibition of thrombin}

The enzymic amidolytic method for determining inhibition of thrombin was based on the spectrophotometric determination of absorbance of the product formed after amide bond cleavage of a chromogenic substrate in the presence of the enzyme. Measurements were performed in 96-well microtiter plates with a final volume of $200 \mu$ l. Thrombin was tested in a final concentration of $1 \mathrm{NIH}(\mathrm{h})$ with the substrate S-2238 (Chromogenix) at $645 \mu \mathrm{M}$ final concentration in aqueous buffer $(50 \mathrm{mM}$ Tris, $300 \mathrm{mM} \mathrm{NaCl}, 0.1 \%$ PEG; pH 8.4). Changes in absorbance at $405 \mathrm{~nm}$ were monitored (Softmax, Molecular Devices, Nanjing, PR China) upon addition of enzyme both with and without inhibitor present at $37^{\circ} \mathrm{C}$ over $30 \mathrm{~min}$. The $\mathrm{IC}_{50}$ values were determined by fixing the enzyme and substrate concentrations and varying the inhibitor concentration.

\section{Fibrinogen/GPIIb/IIIa ELISA}

Similar to the previous method [13], 96-wells plates were coated with fibrinogen $(10 \mu \mathrm{g} / \mathrm{ml})$, diluted in $0.1 \mathrm{M}$ sodium carbonate, $\mathrm{pH} 9.5$ at $4{ }^{\circ} \mathrm{C}$ overnight. After unbound proteins were removed by washing with TACTS (20 mM Tris, $0.15 \mathrm{M} \mathrm{NaCl}$, pH 7.5, $2 \mathrm{mM} \mathrm{CaCl}$, 0.05\% Tween 20), wells were blocked with $1 \%$ bovine serum albumin (BSA) in TACTS for $1 \mathrm{~h}$. The purified human GPIIb/IIIa $(50 \mu \mathrm{l}, 20 \mu \mathrm{g} / \mathrm{ml}$ in TACTS; Enzyme Research Laboratories, IN, USA) were added to the wells; then, samples were added. After $2 \mathrm{~h}$ incubation, wells were washed three-times with TACTS followed by addition of goat antihuman integrin $\beta 3$ antibody (1:2000, CBL479; Millipore, MA, USA) in TACTS containing 0.5\% BSA. Following 1-h incubation at $37^{\circ} \mathrm{C}$, wells were washed (TACTS) and antigoat IgG conjugated alkaline phosphatase (1:1000, A3562; Sigma, Shanghai, PR China) in TACTS containing $0.5 \%$ BSA was added to the wells. The $p$-nitrophenyl phosphate liquid substrate (N7653; Sigma) was added and incubated for $30 \mathrm{~min}$. The reaction was stopped with $3 \mathrm{M} \mathrm{NaOH}$, and absorbance was read at $405 \mathrm{~nm}$ using a Thermomax microplate reader (Molecular Devices). All experiments were performed in triplicate.

\section{Arteriovenous shunt thrombosis model in rat}

Sprague Dawley (SD) rats (both sexes, 180-220 g) were used for as rat arteriovenous shunt thrombosis model according to the previous method [13]. Rats were treated by tail vein injection administration of $1 \mathrm{mg} / \mathrm{kg}$ tirofiban, $10 \mathrm{mg} / \mathrm{kg} \mathrm{LX} 2421$ and 3, 5 and $10 \mathrm{mg} / \mathrm{kg}$ 9-2p. After $6 \mathrm{~h}$ administration, rats were anesthetized by intraperitoneal 


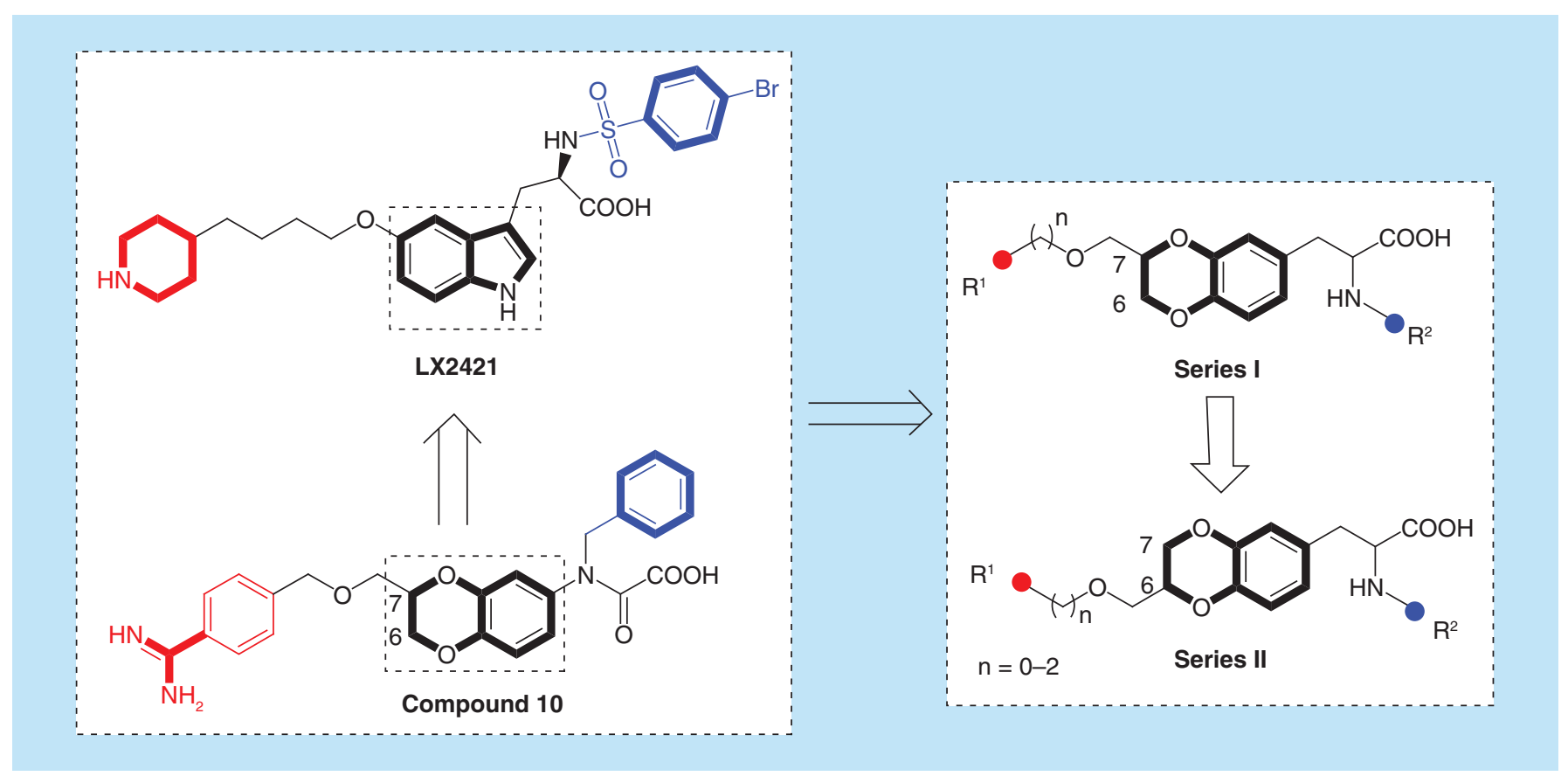

Figure 1. Two novel series of 1,4-benzodioxine derivatives derived from LX2421 and compound 10.

administration of $10 \%$ chloral hydrate $(3 \mathrm{ml} / \mathrm{kg})$. The left jugular vein and right common carotid artery were isolated and catheterized by a shunt catheter (American Health \& Medical Supply International Corp., NY, USA). This catheter is composed of three parts including two 4-cm-long polyethylene (PE) 60 catheters, which were introduced into the blood vessels, and a 12-cm long PE160 catheter that is in the middle of the two PE60 catheters. A rough silk thread (10 cm in length) was in the middle PE160 catheter to induce thrombosis. The shunt was opened for $20 \mathrm{~min}$ and then closed. Silk strings were removed from the middle PE160 catheter; then, the dry weight of thrombus was weighed by drying the thrombus at $60^{\circ} \mathrm{C}$ for $20 \mathrm{~min}$ and removing the cotton thread weigh.

\section{Docking}

The binding modes were generated by CDOCKER in Discovery Studio 3.0 (Accelrys Software, Inc., Nanjing, PR China). In CDOCKER, random ligand conformations were generated through molecular dynamics, and a variable number of translations/rotations were applied to each conformation to generate low-energy orientations of the ligand within the active site of rigid receptor. Final ligand conformations were sorted by CHARMm energy (interaction energy plus ligand strain). The crystal structures of GPIIb/IIIa (PDB entry code: 3ZDX) were extracted from the Protein Database. All ligands were docked in all possible stereoisomeric forms in an active site located sphere with $12 \AA$ A radius for GPIIb/IIIa. The conformers with the lowest CHARMm energy were chosen for interpreting the docking results within 25 docking results.

\section{Result \& discussion}

On the base of our previous research of tryptophan derivatives as platelet aggregation inhibitors derived from natural peptide pENW, LX2421 was identified, which showed potently ADP-induced antiplatelet activity and moderate GPII/IIIa inhibitory activity (as effective as the RGDs [ARG-GLY-ASP-SER], a classical GPII/IIIa antagonist) without increasing risk of bleeding [13,14]. In our further study, we confirmed that indole structure was an unoptimal fragment for antiplatelet activity by reconstructing naphthalene nucleus [15]. In 2013, Kikelj and colleagues reported 1,4-benzodioxine derivatives. Among them, compound $\mathbf{1 0}$ proved to be a significant antithrombotic agents through balancing thrombin inhibitory and GPII/IIIa inhibitory activity [16]. In this communication, we introduced 1,4benzodioxine as an novel scaffold to replace the indole structure within LX2421, which thereby reconstructed novel 1,4-benzodioxine derivatives as platelet aggregation inhibitors (Figure 1). The goal of our investigation is that improves the antiplatelet activity by mixing the basic structure of LX2421 with compound 10. Our study mainly 

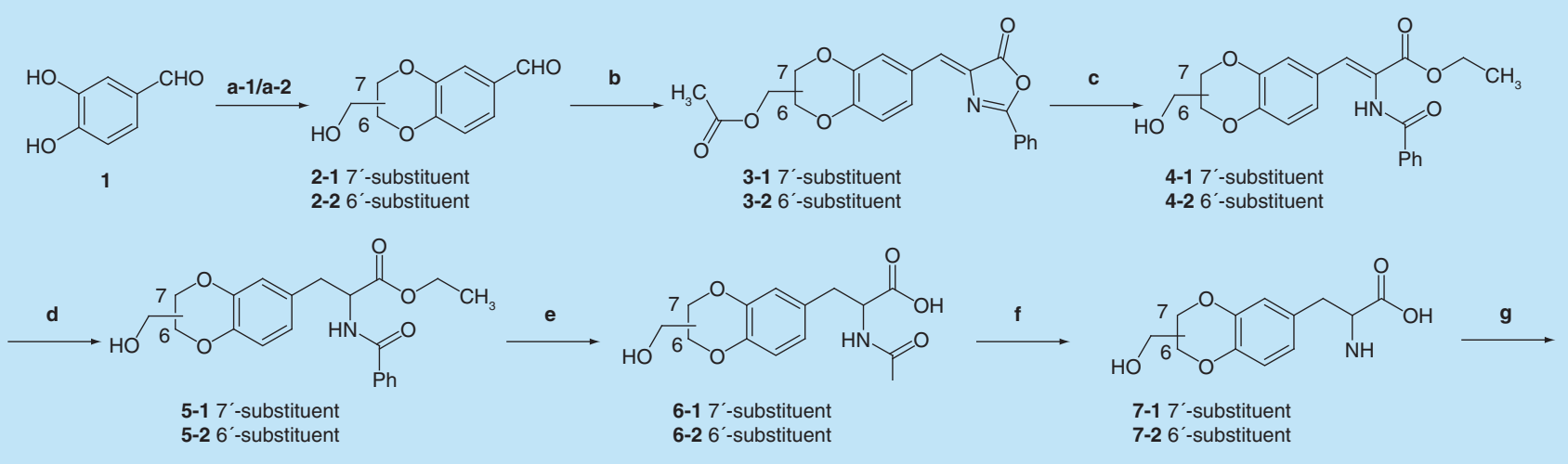

$\mathrm{Ph}$
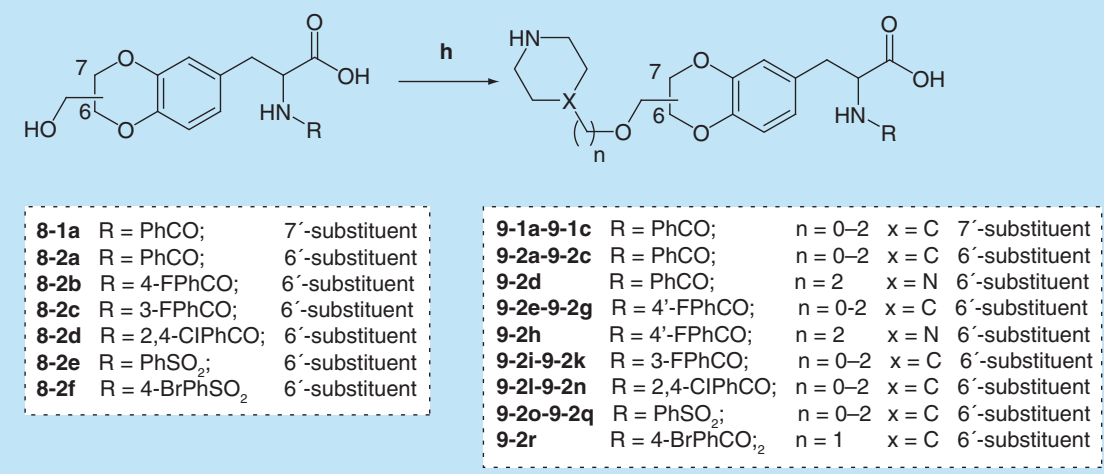

Figure 2. The synthesis route, reagents and conditions of our targeted compounds. Reagents and conditions: (a-1) Epichlorohydrin, $\mathrm{NaHCO}_{3}, \mathrm{DMF}^{\circ} 60^{\circ} \mathrm{C}, 12 \mathrm{~h}$; (a-2) Epichlorohydrin, $\mathrm{NaH}, \mathrm{DMF}, 80^{\circ} \mathrm{C}, 12 \mathrm{~h}$; (b) Hippuric acid, $\mathrm{NaOAc}, \mathrm{Ac}_{2} \mathrm{O}, 100^{\circ} \mathrm{C}, 5 \mathrm{~min} ;(\mathrm{c}) \mathrm{EtONa}, \mathrm{EtOH}$ 0-5 ${ }^{\circ} \mathrm{C}, 4$ h; (d) $\mathrm{H}_{2}, \mathrm{Pd} / \mathrm{C}$, r.t., 12 h; (e) $2 \mathrm{M} \mathrm{NaOH}$, EtOH, r.t. 5 h; (f) $6 \mathrm{~N} \mathrm{HCl}$, reflux, 12 h; (g) $\mathrm{RCl}, \mathrm{K}_{2} \mathrm{CO}_{3}$, acetone $/ \mathrm{H}_{2} \mathrm{O}, 60^{\circ} \mathrm{C}, 12$ h; (h) 1-Boc-4-(2-((methylsulfonyl)oxy)propyl) piperidine or 1-Boc-4-(1-((methylsulfonyl)oxy)propyl) piperidine or 1-Boc-4-(2-((methylsulfonyl)oxy)propyl)piperidine or 1-Boc-4-(2-((methylsulfonyl)oxy)propyl) piperazine, $\mathrm{K}_{2} \mathrm{CO}_{3}, \mathrm{DMF} 80^{\circ} \mathrm{C}, 12 \mathrm{~h}$; $\mathrm{EA}$, $\mathrm{HCl}(\mathrm{g}), 0^{\circ} \mathrm{C}, 6 \mathrm{~h}$.

DMF: Dimethyl formamide; EA: Ethyl acetate; r.t.: Room temperature.

focuses on that: synthesis two novel series of 1,4-benzodioxine derivatives and explore the impact of substituted position of basic linker group, basic group, length of basic linker and $\alpha$-amino substituent on antiplatelet activity (Figure 1); evaluates for the ability to inhibit platelet aggregation induced by two important agonists, ADP and thrombin; confirms the inhibitory activity against two critical proteins in platelet activation, GPII/IIIa and thrombin; and evaluates for the ability to reduce the formation of thrombus in vivo.

The synthesis of our targeted compounds was outlined in Figure 2, reaction of compound $\mathbf{1}$ with epichlorohydrin using $\mathrm{Na}_{2} \mathrm{CO}_{3}$ at $60^{\circ} \mathrm{C}$ gave compound $\mathbf{2}-\mathbf{1}$, while the reaction of compound $\mathbf{1}$ with epichlorohydrin using $\mathrm{NaH}$ at $80^{\circ} \mathrm{C}$ gave compound $\mathbf{2 - 2}$ [15]. Compounds $\mathbf{2 - 1 / 2}$ were cyclized with hippuric acid to afford intermediates 3-1/2. Then, compounds $\mathbf{4}-\mathbf{1 / 2}$ were prepared from $\mathbf{3}-\mathbf{1 / 2}$ by hydrolyzation of lacton and acetate. Reduction of $\mathbf{4}-\mathbf{1} / \mathbf{2}$ with $\mathrm{Pd} / \mathrm{C}$ gave compounds $\mathbf{5}-\mathbf{1 / 2}$, which were further hydrolyzed with EtONa to provide compounds 6-1/2, subsequent treatment with $6 \mathrm{~N} \mathrm{HCl}$ under refluxing gave intermediates $7-\mathbf{1} / \mathbf{2}$. Compounds $\mathbf{7 - 1 / 2}$ were further reacted with various acyl chloride or sulfonyl chloride, affording compounds 8-1a and $\mathbf{8}-\mathbf{2 a}-\mathbf{f}$. Then, final compounds 9-1a-c and 9-2a-r were achieved upon etherification with 4-piperidinyl/piperazinyl containing methyl sulfonic acid ester and deprotection of Boc (see the Supplementary Information for details).

Our targeted compounds, first were assayed for their antiplatelet aggregation activity using human PRP induced by ADP $(10 \mu \mathrm{M})$ agonist at $50 \mu \mathrm{M}$. Unexpectedly, compounds 9-1a-c displayed negative activity with the C-linker attaching piperidyl group at 7 th position of the scaffold and benzoyl substituent at the $\alpha$-amino. Interestingly, the antiplatelet activities of $\mathbf{9 - 2 a - c}$ were significantly enhanced by changing linker of the 7 th position to the 6th position. Meanwhile, compounds $\mathbf{9 - 2} \mathbf{b}$ containing one C-linker was more potent than zero C-linker contained 9-2a and two C-linker contained 9-2c as shown in Table 1. The result indicated that one C-linker attaching 


\section{Table 1. Biological activity of 1,4-benzodioxine derivatives: inhibition of platelet aggregation induced by ADP and}

\section{thrombin at $50 \mu \mathrm{M}$.}

\begin{tabular}{|c|c|c|c|c|c|c|}
\hline \multirow[t]{2}{*}{ Cpd } & \multirow[t]{2}{*}{ Substituent } & \multirow[t]{2}{*}{$\mathrm{R}^{1}$} & \multirow[t]{2}{*}{$\mathbf{R}^{2}$} & \multirow[t]{2}{*}{ n } & \multicolumn{2}{|c|}{ Inhibition \% $(50 \mu \mathrm{M})^{\ddagger}$} \\
\hline & & & & & ADP & Thrombin \\
\hline 9-1a & 7 & & & 0 & $<5$ & $<5$ \\
\hline $9-1 c$ & 7 & & & 2 & $<5$ & $<5$ \\
\hline $9-2 b$ & 6 & & & 1 & $38.6 \pm 0.5$ & $11.9 \pm 1.5$ \\
\hline $9-2 c$ & 6 & & & 2 & $15.8 \pm 2.1$ & $86.2 \pm 1.7$ \\
\hline $9-2 d$ & 6 & & & 2 & $7.2 \pm 1.4$ & $82.7 \pm 1.2$ \\
\hline $9-2 e$ & 6 & & & 0 & $7.9 \pm 1.3$ & $17.2 \pm 0.5$ \\
\hline $9-2 f$ & 6 & & & 1 & $29.3 \pm 0.6$ & $37.3 \pm 1.2$ \\
\hline $9-2 g$ & 6 & & & 2 & $20.1 \pm 1.6$ & $85.1 \pm 1.8$ \\
\hline $9-2 \mathrm{~h}$ & 6 & & & 2 & $16.9 \pm 1.4$ & $74.1 \pm 1.9$ \\
\hline
\end{tabular}


Table 1. Biological activity of 1,4-benzodioxine derivatives: inhibition of platelet aggregation induced by ADP and

\section{thrombin at $50 \mu \mathrm{M}$ (cont.).}

Cpd

Substituent

$\mathrm{R}^{1}$

$\mathrm{R}^{2}$

Inhibition \% $(50 \mu \mathrm{M}) \ddagger$

ADP

Thrombin

9-2i<smiles>CC(=O)c1cccc(F)c1</smiles>

$25.5 \pm 0.9$

$65.5 \pm 2.1$

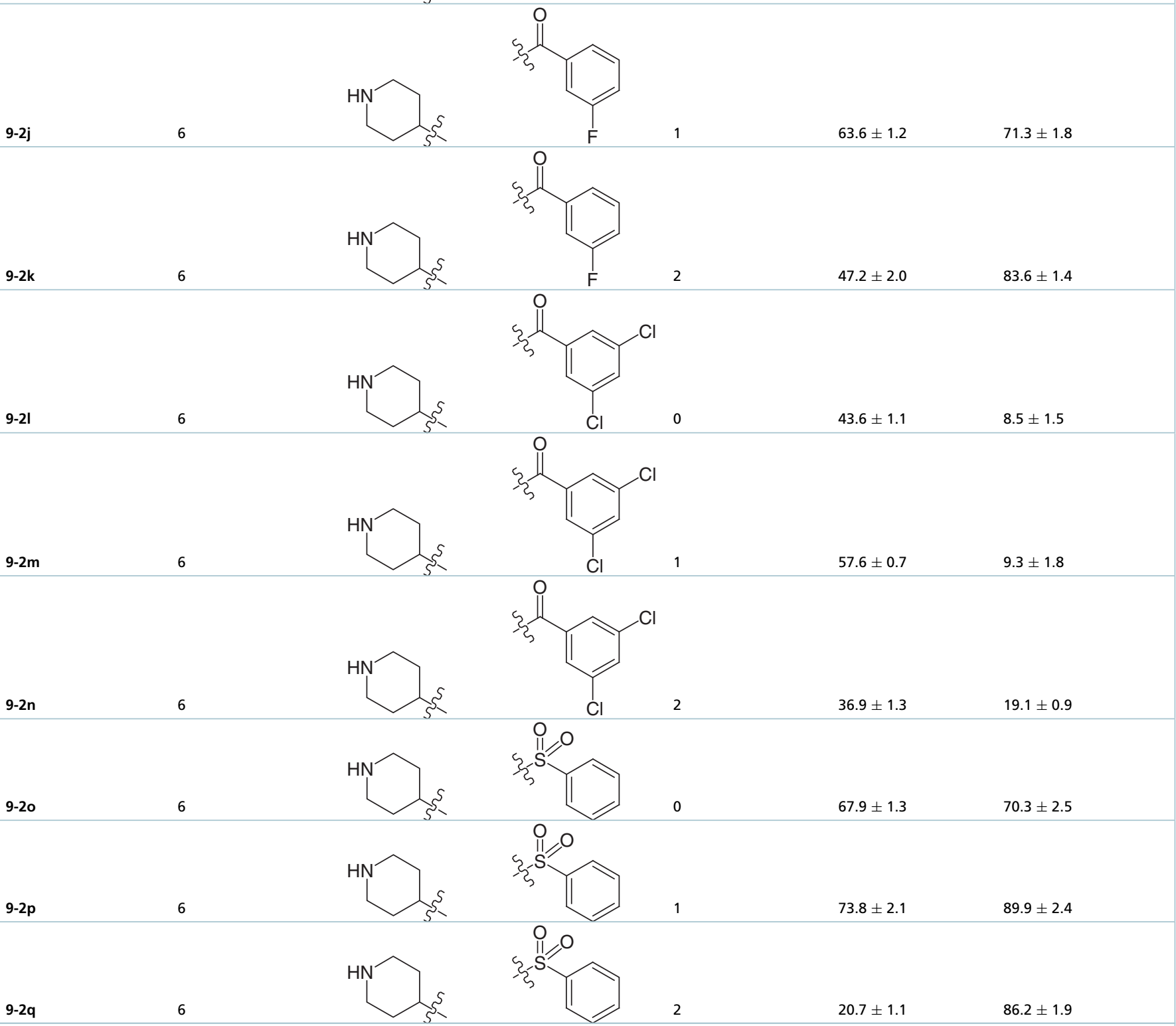

$\dagger$ In vitro inhibition of ADP-induced $(10 \mu \mathrm{M})$ human platelet aggregation; see the supporting information for details; the data represent the mean of at least three independent determinations.

$\ddagger$ In vitro inhibition of thrombin-induced $(0.33 \mathrm{U})$ human platelet aggregation; see the supporting information for details; values are means from two to three independent dose-response curves. 


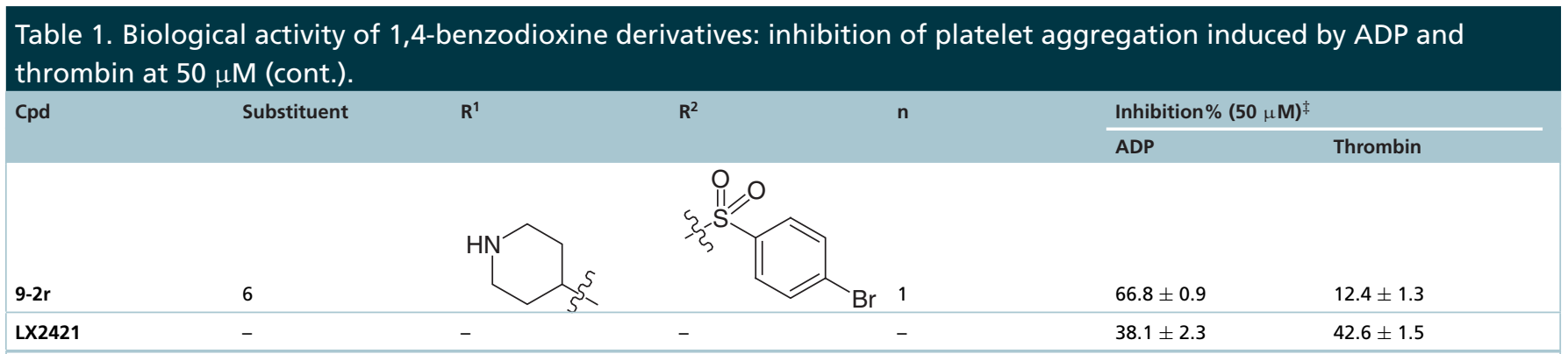

$\dagger / n$ vitro inhibition of ADP-induced $(10 \mu \mathrm{M})$ human platelet aggregation; see the supporting information for details; the data represent the mean of at least three independent determinations.

$\ddagger$ In vitro inhibition of thrombin-induced $(0.33 \mathrm{U}$ ) human platelet aggregation; see the supporting information for details; values are means from two to three independent dose-response curves.

the basic groups was favorable for the antiplatelet activity. Interestingly, following the change of the substituent at the $\alpha$-amino, the one C-linker was still most suitable. Compounds 9-2d and 9-2h containing piperazine group were synthesized to explore the impact of basic group on the antiplatelet activity in comparison with their corresponding compounds 9-2c and 9-2g. The result demonstrated that contained piperidyl compounds were more potent than contained piperazine compounds (9-2d vs 9-2c, 9-2h vs 9-2g), indicating that piperidyl was a more suitable basic group for our compounds. In view of our previous investigation, addition of halogen atom such as fluoro and bromine to the phenyl ring of the substituent at the $\alpha$-amino could improve the antiplatelet activity. Therefore, contained fluoro atom compounds 9-2e-k and contained chlorine atom compounds 9-21-n were synthesized. Unexpectedly, most of compounds were just showed comparable activity or slight enhanced activity as the corresponding compounds. To further increase the activities of our compounds, benzene sulfonyl substituent was introduced to replace the benzoyl substituent on the basis of our previous structure-activity relationship (SAR) summary. The activities of contained benzenesulfonyl compounds $\mathbf{9 - 2 0}-\mathbf{p}$ were significantly enhanced, especially 9-2p, with the platelet aggregation inhibition ratio of $73 \%$. Similarly, introduction of halogen such as bromine atom into the phenyl ring of benzenesulfonyl group (9-2r) had no remarkable effect on the activity. Moreover, to confirm the necessity of basic group for antiplatelet activity, we also test the antiplatelet activity of $\mathbf{8}-\mathbf{2 a}-\mathbf{f}$ with the ADP inducer. The result showed that 8-2a-f displayed negative antiplatelet activity, suggesting that the basic groups are critical fragments in our compounds for antiplatelet activity, which may interact with potential target.

Besides ADP pathway, thrombin pathway is another important signaling to active platelet aggregation. Therefore, our compounds were further assayed for the antiplatelet activity using human PRP induced by thrombin $(0.33 \mathrm{U})$ agonist at $50 \mu \mathrm{M}$. As shown in Table 1, moderate to good inhibition ratio $(>60 \%)$ was observed for most of compounds, indicating that our compounds could inhibit the platelet aggregation by blocking two different agonists mediated signaling pathway. In this assay, the length of C-linker also played a critical role in antiplatelet activity. Different from the SAR based on ADP-induced antiplatelet activity, the most suitable C-linker for thrombin-induced antiplatelet activity was two-carbon linker. For the basic groups at the tail of linker and the substituents at the $\alpha$-amino, the piperidyl group and the benzene sulfonyl group were still the most favorable. Considering dual activity of inhibiting ADP and thrombin-induced pathway, 9-2p, more potent than LX2421, with the inhibition ratio of 73.3 and $89.9 \%$, respectively, was selected to be evaluated for the antiplatelet activity using concentration-inhibition curve ex vivo.

Further study, 9-2p was detailedly evaluated for the antiplatelet activity using human PRP induced by ADP $(10 \mu \mathrm{M})$ and thrombin $(0.33 \mathrm{U})$ agonist in comparison with lead compound LX2421. As shown in Figure 3, in the ADP-induced antiplatelet aggregation assay, 9-2p showed the $\mathrm{IC}_{50}$ value of $41.7 \mu \mathrm{M}$, two-times more potent than LX2421. In the thrombin-induced antiplatelet aggregation assay, the $\mathrm{IC}_{50}$ value of $\mathbf{9 - 2} \mathbf{p}$ is $22.2 \mu \mathrm{M}$, three-times more potent than LX2421. These results further indicated that 9-2p can significant inhibit platelet aggregation induced by ADP and thrombin.

To explore whether 9-2p directly targets on the final common signaling protein, GPII/IIIa receptor, to block the two ADP and thrombin-induced signaling pathways, compound 9-2p was also evaluated for the binding affinity to GPIIb/IIIa receptor in the fibrinogen/GPIIb/IIIa ELISA. As shown in Figure 4A, compound 9-2p displayed potently GPII/IIIa antagonistic activity with the $\mathrm{IC}_{50}$ value of $2.3 \mu \mathrm{M}$, two-times more potent than classical GPIIb/IIIa antagonist, RGDs. In addition, considering the dual mechanism of compound $\mathbf{1 0}$ reported 

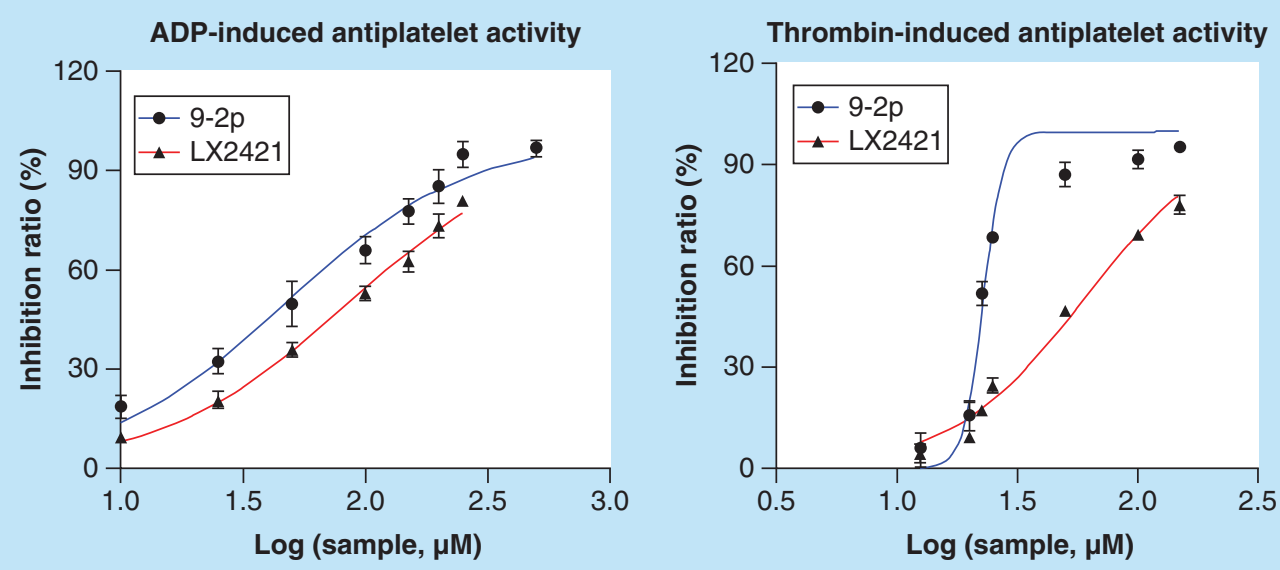

Figure 3. The antiplatelet aggregation activity of LX2421 and 9-2p induced by ADP at the concentration of 10, 25, $50,100,150,200,250,300 \mu \mathrm{M}$ and by thrombin at the concentration of 12.5, 20, 22.5, 25, 50, 100, $200 \mu \mathrm{M}$. The activity was shown as an inhibition ratio to control. Data are expressed as mean \pm SD $(n=3)$. See the Supplementary Information for details.

(A)

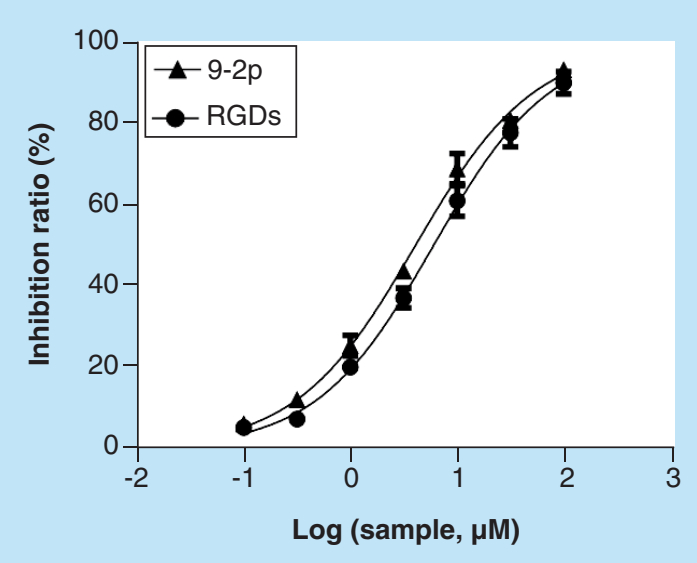

(B)

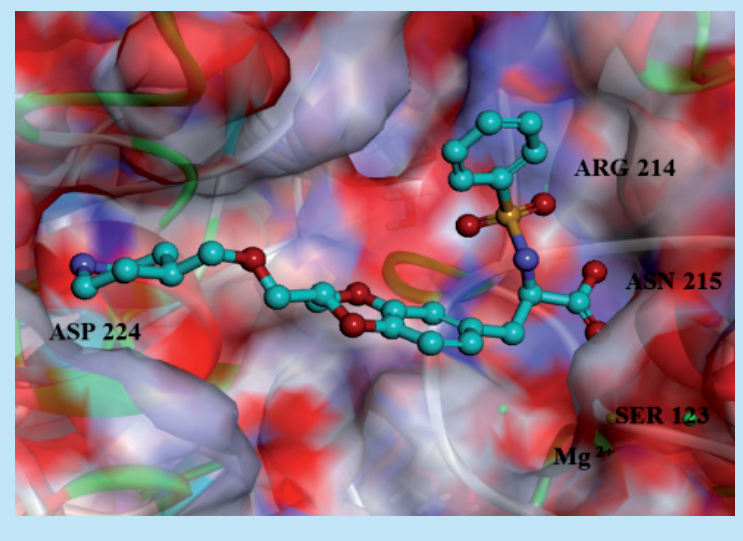

Figure 4. The GPIIb/IIla inhibitory activity of 9-2p and the binding mode between GPIlb/IIla and 9-2p. (A) The GPIIb/IIla receptor inhibitory activities of 9-2p in comparison with RGDs in the fibrinogen/GPIIb/IIla ELISA. Data are expressed as mean \pm SD $(n=3)$; (B) Docking mode of 9-2p binding to GPIIb/llla receptor. See the Supplementary Information for details.

by Kikelj and colleagues, compound $\mathbf{9 - 2} \mathbf{p}$ was further evaluated for the thrombin inhibitory activity. Interestingly, compound 9-2p showed negative inhibitory activity against thrombin $\left(\mathrm{IC}_{50}>100 \mu \mathrm{M}\right)$, suggesting that $\mathbf{9 - 2} \mathbf{p}$ selectively inhibits GPII/IIIa receptor to provide the ability to inhibit platelet aggregation.

Subsequently, a docking study of 9-2p with GPII/IIIa was performed using the cDOCKER in Discovery Studio 3.0 software. In order to explore its binding model against GPII/IIIa, the crystal complex of RGD with GPII/IIIa (PDB:3ZDX) was selected for the docking study. The top 25-ranked docking poses of $\mathbf{9 - 2} \mathbf{p}$ were generated and the best superimposition was shown in Figure 4B. The model revealed that the carboxyl group in $\mathbf{9 - 2} \mathbf{p}$ forms hydrogen bond with Ser123, Asn215 residue and interacts with the MIDAS $\mathrm{Mg}^{2+}$ ion of GPIIla, the sulfamide group in compound 9-2p exhibits hydrogen bond with Arg214 residues and the 4-piperidyl group in both has slat bridge with Asp224 residue of GPIIb. The result indicated that $\mathbf{9 - 2} \mathbf{p}$ was situated in the active site of GPII/IIIa.

Compound $9-2 \mathbf{p}$ has further been evaluated for its antithrombotic activity in vivo in comparison with approved GPIIb/IIIa antagonist tirofiban and LX2421. In the rat arteriovenous shunt thrombosis model, SD rats were 
Figure 5. The antithrombotic activities of $9-2 p$ in vivo in the rat arteriovenous shunt thrombosis model in comparison with LX2421 and tirofiban. Three doses were tested for $9-2 \mathrm{p}(3,5$ and $10 \mathrm{mg} / \mathrm{kg})$, one dose $(10 \mathrm{mg} / \mathrm{kg})$ was tested for LX2421 and one dose $(1 \mathrm{mg} / \mathrm{kg})$ was tested for tirofiban. Data are expressed as mean \pm SD $(n=6)$.

${ }^{*} \mathrm{p}<0.05, * * * * \mathrm{p}<0.0001$ compared with control group. See the Supplementary Information for details.

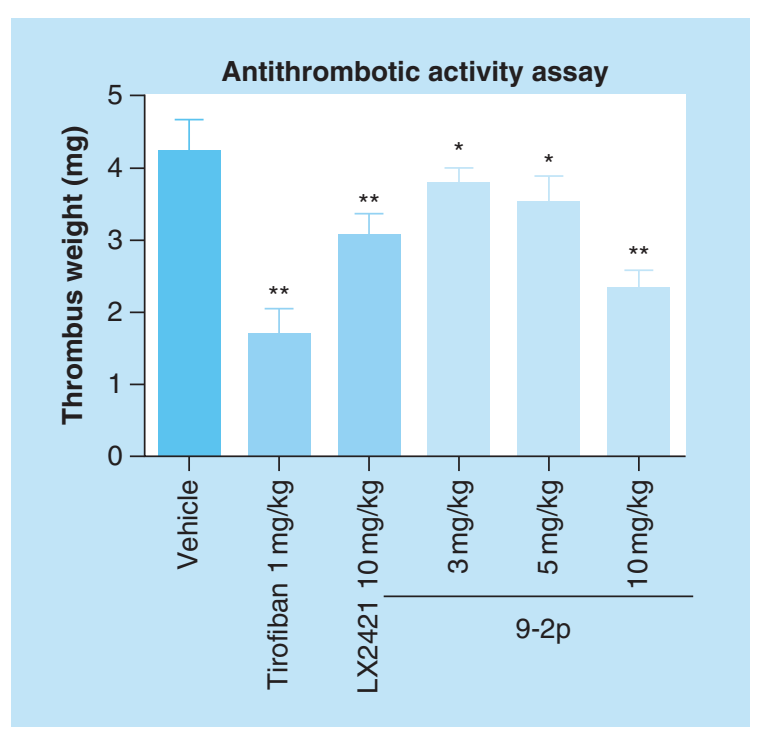

randomly divided into six groups with six rats (males and females in half) in each group and injected intravenously with 9-2p $(3,5,10 \mathrm{mg} / \mathrm{kg})$, tirofiban $(1 \mathrm{mg} / \mathrm{kg}), \mathbf{L X 2 4 2 1}(10 \mathrm{mg} / \mathrm{kg})$ or vehicle. The weights of dry thrombus were measured $6 \mathrm{~h}$ later at room temperature. As shown in Figure 5, compound 9-2p can significantly reduce the formation of the thrombus in a dose-dependent manner. At $10 \mathrm{mg} / \mathrm{kg}, \mathbf{9 - 2} \mathbf{p}$ decreases the experimental thrombosis by $44.3 \%$, more effective than $\mathbf{L X 2 4 2 1}$ at $10 \mathrm{mg} / \mathrm{kg}$ and slight less effective than tirofiban at $1 \mathrm{mg} / \mathrm{kg}$. The result indicated that 9-2p is a promising antithrombotic agent being further evaluated for the risk of bleeding and the mechanism of antiplatelet aggregation.

\section{Conclusion}

In summary, two novel series of 1,4-benzodioxine derivatives were designed and synthesized on the basis of the previous antiplatelet agents LX2421 and compound 10. The structure-activity relationship was studied following the assay of antiplatelet aggregation activity induced by ADP and thrombin. These efforts led to the identification of the most potent compound $\mathbf{9 - 2} \mathbf{p}$. In the ADP/thrombin-induced antiplatelet aggregation activity assay, 9-2p showed the $\mathrm{IC}_{50}$ values of 41.7 and $22.2 \mu \mathrm{M}$, respectively, more potent than LX2421. Compound 9-2p displayed GPII/IIIa antagonistic activity with the $\mathrm{IC}_{50}$ value of $2.3 \mu \mathrm{M}$, as potent as RGDs and without thrombin inhibitory activity. Moreover, compound 9-2p can significantly reduce the formation of thrombus in the rat arterio-venous shunt thrombosis model, more effective than LX2421. However, compound 9-2p showed weaker antiplatelet and antithrombotic activity in comparison with our previous naphthyl derivative, compound LX14 [14], demonstrating that 1,4-benzodioxine was not as promising as the corresponding naphthyl scaffold. The reason may be is that the conjugation between naphthalene nucleus and Tyr190 enhances the binding of LX14 and GPIIb/IIIa markedly (Figure 6). Then, compound 9-2p could be a lead platelet aggregation inhibitor for further optimization by improving the conjugation between 1,4-benzodioxine core and Tyr190.

\section{Future perspective}

Despite oral administration of aspirin and clopidogrel, the approved GPIIb/IIIa antagonists, such as tirofiban and eptifibatide, were still administrated intravenously. Up to now, some oral GPIIb/IIIa antagonists (e.g., roxifiban) had been entered into Phase III in clinic, however, none of them was approved for treatment of thrombotic disorders with side effects including severe bleeding risk, thrombocytopenia and increasing patient mortality. The development of oral GPIIb/IIIa antagonists has been the most challenging problem in the field of GPIIb/IIIa antagonist research, which should balance the antithrombotic efficacy and side effects (especially risk of bleeding). In our work, we found some promising platelet aggregation inhibitors targeting GPIIb/IIIa, which was evaluated for their antithrombotic activity through intravenous administration in comparison with tirofiban. We will further test and improve oral bioavailability and pharmacokinetic properties of our compounds by eliminating the basic group or developed oral GPIIb/IIIa antagonists directly based on lead compounds RUC-1/4 reported in recent years. 

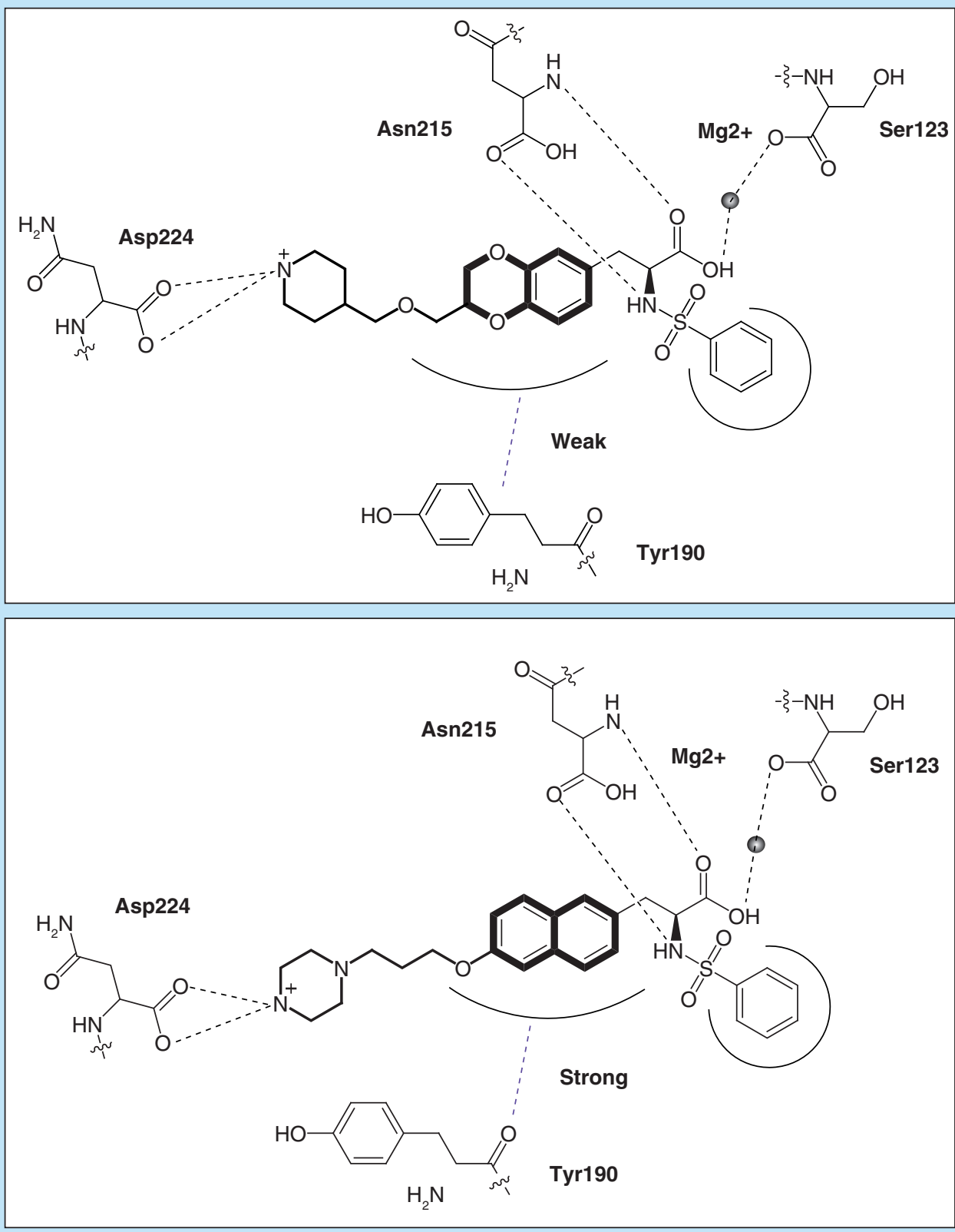

Figure 6. The 2D-binding modes of 9-2p and LX14 with GPIIb/IIla.

Antiplatelet drugs displayed critical role in the treatment of arterial thromboembolic diseases. However, kinds of side effects, such as antiplatelet resistance and risk of bleeding seriously affected drug application in clinic. In the future, to reduce these limitations, novel antiplatelet therapy will be researched by targeting new platelet surface receptors or critical proteins at the inside signaling pathway. Platelet surface receptors of PAR-4, GPIb-IX-V and P-selectin were identified as promising targets to decrease the risk of bleeding in recent years. In addition, novel antiplatelet agents will also be developed by structure-based drug design or screening from natural peptides like snake venom. Some natural novel antithrombotic compounds with lower bleeding risk will be studied for their mechanisms, which is a rapid method to find promising antiplatelet targets.

For the classical targets like GPIIb/IIIa, P2Y and COX-1, the corresponding novel antagonists will be developed following the deepen research of their specific mechanism. We hope that these novel antagonists will display favorable efficacy and safety in clinical trial. 


\section{Executive summary}

- Antiplatelet therapies have displayed an important role in the treatment of cardiovascular arterial disease.

- Though antiplatelet therapy has remarkably reduced the risk of recurrent ischemic events in patient, there remains a substantial incidence of arterial thrombosis in patients because of current therapy limitations including antiplatelet resistant, bleeding risk and thrombocytopenia.

- Novel 1,4-benzodioxine derivatives were designed by mixing the structures of LX2421 and compound 10.

- Two novel series of 1,4-benzodioxine derivatives were synthesized and evaluated for the ability to inhibit platelet aggregation as well as the summary of SAR.

- The most potent compound, 9-2p was identified and showed significant antiplatelet activity with the $\mathrm{IC}_{50}$ values of 41.7 and $22.2 \mu \mathrm{M}$ induced by ADP and thrombin, respectively, more potent than that of LX2421.

- Compound 9-2p exhibited GPIIb/IIla antagonistic activity with the IC $\mathrm{C}_{50}$ value of $2.3 \mu \mathrm{M}$, as potent as RGDs with no thrombin inhibitory activity.

- In vivo study showed compound 9-2p displayed remarkable antithrombotic activity, more effective than LX2421 but less effective than tirofiban.

- As a novel antiplatelet compound, Compound 9-2p could be a lead compound for further study.

Financial \& competing interests disclosure

This work was supported by the National Natural Science Foundation of China (21672050), China Pharmaceutical University and Hefei University of Technology. The authors have no other relevant affiliations or financial involvement with any organization or entity with a financial interest in or financial conflict with the subject matter or materials discussed in the manuscript apart from those disclosed.

No writing assistance was utilized in the production of this manuscript.

\section{References}

Papers of special note have been highlighted as: $\bullet$ of interest; $\bullet \bullet$ of considerable interest

1. Hagemeyer CE, Peter K. Targeting the platelet integrin GPIIb/IIIa. Curr. Pharm. Design 16, 4119-4133 (2010).

- Provides the functional mechanism of GPIIb/IIIa at the pathway of platelet aggregation.

2. Xie ZL, Cao C, Feng S et al. Progress in the research of GPIIb/IIIa antagonists. Future Med. Chem. 7, 1149-1171 (2015).

- Excellent review on the progress in the research of GPIIb/IIIa antagonists.

3. Karolina A, Dimitrios A. Use of antiplatelet agents in sepsis: a glimpse into the future. Thromb. Res. 133, 131-138 (2014).

4. Alan DM. Antiplatelet therapies for the treatment of cardiovascular disease. Nat. Rev. drug. Discov. 9, 154-169 (2010).

5. Andrei AK, Georgiy VS, Pavel GP et al. Synthesis, biological evaluation, x-ray molecular structure and molecular docking studies of RGD mimetics containing 6-amino-2,3-dihydroisoindolin-1-one fragment as ligands of integrin $\alpha \operatorname{IIb} \beta 3$. Bioorg. Med. Chem. 21, 4646-4661 (2013).

- Provides the rational design for novel GPIIb/IIIa antagonists.

6. Trstenjak U, Ila J, Kikelj D. Low molecular weight dual inhibitors of factor Xa and fibrinogen binding to GPIIb/IIIa with highly overlapped pharmacophores. Eur. J. Med. Chem. 64, 302-313 (2013).

- Provides the rational design for novel dual inhibitors of factor Xa and GPIIb/IIIa.

7. Paul CA, Karlheinz P. GPIIb/IIIa inhibitors: from bench to bedside and back to bench again. Thromb. Haemostasis 107, 808-814 (2012).

-. Provides the functional mechanism of GPIIb/IIIa at the pathway of platelet aggregation.

8. Ana N, Jihong L, Sarasija N et al. Structure-based virtual screening of small-molecule antagonists of platelet integrin $\alpha$ IIb $\beta 3$ that do not prime the receptor to bind ligand. J. Comput. Aided Mol. Des. 26, 1005-1015 (2012).

9. Tian X, Wang L, Xia S et al. Synthesis of $2 \mathrm{H}$-benzo[ b] [1,4] oxazin-3(4 H)-one derivatives as platelet aggregation inhibitors. Bioorg. Med. Chem. Lett. 22, 204-206 (2012).

10. Chang L, Jia LT, Si YX et al. 1,4-Benzoxazine-3(4H)-ones as potent inhibitors of platelet aggregation: design, synthesis and structure-activity relations. Chem. Pharm. Bull. 62, 915-920 (2014).

11. Udaya ST, Martin G, Fang L et al. Resistance to antiplatelet drugs: what progress has been made. Expert Opin. Pharmacother. 15, 2553-2564 (2014).

-. Provides the new progress of antiplatelet drugs.

12. Ding $\mathrm{X}$, Liu $\mathrm{T}, \mathrm{Xie} \mathrm{Z}$ et al. A naphthalenic derivative ND-1 inhibits thrombus formation by interfering the binding of fibrinogen to integrin $\alpha \operatorname{IIb} \beta_{3}$. Biomed. Res. Int. doi:10.1155/2016/8587164 (2016). 
13. Xiong J, Bai L, Fang W et al. New peptide pENW (pGlu-Asn-Trp) inhibits platelet activation by attenuating Akt phosphorylation. Eur. J. Pharm. Sci. 45, 552-558 (2012).

14. Xie ZL, Feng S, Wang Y et al. Design, synthesis of novel tryptophan derivatives for antiplatelet aggregation activity based on tripeptide pENW (pGlu-Asn-Trp). Eur. J. Med. Chem. 102, 363-374 (2015).

15. Xie ZL, Oscar B, Zhao LL et al. Design, synthesis and evaluation of novel 2-amino-3-(naphth-2-yl)propanoic acid derivatives as potent inhibitors of platelet aggregation. Eur. J. Med. Chem. 125, 197-209 (2017).

-. Provides scaffold of 2-amino-3-(naphth-2-yl)propanoic acid to design novel antiplatelet agents.

16. Ilic M, Dunkel P, Ilas J et al. Towards dual antithrombotic compounds- balancing thrombin inhibitory and fibrinogen GPIIb/IIIa binding inhibitory activities of 2,3-dihydro-1,4-benzodioxine derivatives through regio- and stereoisomerism. Eur. J. Med. Chem. 62, 329-340 (2013).

-• Provides scaffold of 1,4-benzodioxine to design dual inhibitors of thrombin and GPIIb/IIIa. 